\title{
Double-occupancy errors, adiabaticity, and entanglement of spin qubits in quantum dots
}

\author{
John Schliemann, ${ }^{1,2}$ Daniel Loss, ${ }^{3}$ and A. H. MacDonald ${ }^{1,2}$ \\ ${ }^{1}$ Department of Physics, Indiana University, Bloomington, Indiana 47405 \\ ${ }^{2}$ Department of Physics, The University of Texas, Austin, Texas 78712 \\ ${ }^{3}$ Department of Physics and Astronomy, University of Basel, Klingelbergstraße 82, CH-4056 Basel, Switzerland
}

(Received 6 September 2000; published 2 February 2001)

\begin{abstract}
Quantum gates that temporarily increase singlet-triplet splitting in order to swap electronic spins in coupled quantum dots lead inevitably to a finite double-occupancy probability for both dots. By solving the timedependent Schrödinger equation for a coupled dot model, we demonstrate that this does not necessarily lead to quantum computation errors. Instead, the coupled dot ground state evolves quasiadiabatically for typical system parameters so that the double-occupancy probability at the completion of swapping is negligibly small. We introduce a measure of entanglement that explicitly takes into account the possibilty of double occupancies and provides a necessary and sufficient criterion for entangled states.
\end{abstract}

DOI: 10.1103/PhysRevB.63.085311

PACS number(s): 85.35.Be, 85.35.Gv, 03.67.- a

\section{INTRODUCTION}

In the past several years there has been a great deal of interest in possible physical realizations of quantum computing bits and operations. ${ }^{1}$ Among the various proposals, solid state systems are particularly attractive since they are more easily integrated into large quantum networks. In particular, semiconductor nanostructures that use the spin degree of freedom of the electrons ${ }^{2}$ (rather than their charge) for information processing are of special interest since they can take advantage of the comparatively long spin coherence times in such materials. ${ }^{3-5}$

A key challenge is the construction of systems composed of two coupled quantum dots that can be coupled to perform swap operations $\mathcal{U}_{S W}$, i.e., unitary two-qubit operations that interchange the spin states (qubits) of the electrons on the two dots. ${ }^{2,6-10}$ By combining the "square root" $\mathcal{U}_{S W}^{1 / 2}$ of such a swap with other isolated-qubit manipulations, one can construct a quantum XOR gate. A quantum XOR gate, along with isolated-qubit operations, has been shown to be sufficient for the implementation of any quantum algorithm. ${ }^{11}$ Hence, a practical and reliable realization of a swap gate would be an important step towards the fabrication of a solid-state quantum computer.

The swap operation of electron spin states in a double dot system can be realized in principle by turning on a timedependent exchange coupling between the spins as a "source of entanglement." In practice the exchange interaction is provided by singlet-triplet splitting in a double dot, which is always accompanied by a finite interdot electron tunneling amplitude.

In a recent work, Das Sarma and co-workers, ${ }^{9,10}$ emphasized that exchange interactions in the range of interest are accompanied by a substantial probability, during the swap operation, that both electrons will be on the same dot. In this paper we demonstrate that, contrary to naive expectations, these virtual double occupancies will not, under circumstances typically envisioned, lead to an important increase in quantum computing errors. Double occupancy is not a fatal problem for quantum dot based quantum computing with spins. The occurrence of double occupancies during the swapping process does not lead to processing errors, provided that the double occupancies are sufficiently suppressed when the swapping of spin states is completed. The principle purpose of the present paper is to illustrate this basic feature within the Hund-Mulliken description of a quantum dot hydrogen molecule. We will see that, in a system of identical dots, the time evolution of this system can be reduced to the problem of a pseudospin half in a time-dependent pseudomagnetic field. In particular, the question of whether double occupancies are a severe obstacle for swap operations in the quantum dot system is equivalent to the question of how close the pseudospin dynamics is to its adiabatic limit. Simple numerical studies presented in Sec. IV show that the pseudospin has an approximately adiabatic time evolution for a ramarkably broad range of coupling ramp times. It turns out that this behavior holds even if the inversion symmetry along the $x$ axis connecting the dots is broken (e.g., in the presence of an electric field).

A secondary purpose of this work is to introduce a coordinate-independent measure of entanglement appropriate for the Hilbert space of the above system. This quantity provides a necessary and sufficient criterion for the entanglement of quantum states. It differs from other entanglement criteria proposed in the literature ${ }^{12}$ in so far as it takes into account states with double occupancies. This generalizes the typical situation of Einstein-Podolsky-Rosen experiments. We expect this measure of entanglement to be useful in the theoretical study of coupled quantum dots (or similar quantum-confined nanostructures), independent of the particular model considered here.

\section{THE MODEL}

We consider a system of two electrons in two laterally coupled quantum dots. The experimental motivation for the model described below has been discussed elsewhere. ${ }^{6-8}$ Here, we just summarize its basic features.

The Hamiltonian is given by $\mathcal{H}=T+C$, where $C$ denotes the Coulomb repulsion between the electrons and $T$ $=\Sigma_{i=1,2} h_{i}$ is the one-particle part with 


$$
h_{i}=\frac{1}{2 m}\left(\vec{p}_{i}+\frac{e}{c} \vec{A}\left(\vec{r}_{i}\right)\right)^{2}+V\left(\vec{r}_{i}\right) .
$$

The one-particle Hamiltonian $h_{i}$ describes electron dynamics confined to the $x y$ plane in a perpendicular magnetc field $B$. The effective mass $m$ is a material-dependent parameter. The coupling of the dots (which includes tunneling) is modeled by a quartic potential

$$
V(x, y)=\frac{m \omega_{0}^{2}}{2}\left[\frac{1}{4 a^{2}}\left(x^{2}-a^{2}\right)^{2}+y^{2}\right],
$$

which separates into two harmonic wells of frequency $\omega_{0}$ (one for each dot) in the limit $2 a \gg 2 a_{0}$, where $a$ is half the distance between the dots and $a_{0}=\sqrt{\hbar / m \omega_{0}}$ is the effective Bohr radius of a dot.

Following Burkard et al., ${ }^{6}$ we employ the Hund-Mulliken method of molecular orbits to describe the low-lying spectrum of our system. This approach concentrates on the lowest orbital states in each dot and is an extension of the Heitler-London method also discussed in Ref. 6. The HundMulliken approach accounts for double occupancies and is therefore useful for investigating the questions at issue here.

In the usual symmetric gauge $\vec{A}=B(-y, x, 0) / 2$ the FockDarwin ground state of a single dot with harmonic confinement centered around $\vec{r}=( \pm a, 0,0)$ reads

$$
\begin{aligned}
\varphi_{ \pm a}(x, y)= & \sqrt{\frac{m \omega}{\pi \hbar}} \exp \left(\frac{m \omega}{2 \hbar}\left\{(x \mp a)^{2}+y^{2}\right\}\right) \\
& \times \exp \left( \pm \frac{i}{2} y \frac{a}{l_{B}^{2}}\right),
\end{aligned}
$$

where $l_{B}=\sqrt{\hbar c / e B}$ is the magnetic length, and the frequency $\omega$ is given by $\omega^{2}=\omega_{0}^{2}+\omega_{L}^{2}$, where $\omega_{L}=e B / 2 m c$ is the usual Larmor frequency. From these nonorthogonal one-particle states, we construct the orthonormalized states $|A\rangle$ and $|B\rangle$ with wave functions

$$
\begin{aligned}
& \langle\vec{r} \mid A\rangle=\frac{1}{\sqrt{1-2 S g-g^{2}}}\left(\varphi_{+a}-g \varphi_{-a}\right), \\
& \langle\vec{r} \mid B\rangle=\frac{1}{\sqrt{1-2 S g-g^{2}}}\left(\varphi_{-a}-g \varphi_{+a}\right),
\end{aligned}
$$

with $S$ being the overlap between the states (3) and $g=(1$ $\left.-\sqrt{1-S^{2}}\right) / S$. For appropriate values of system parameters such as the interdot distance and the external magnetic field, the overlap $S$ becomes exponentially small. ${ }^{6}$ In this limit, an electron in one of the states $|A\rangle,|B\rangle$ is predominantly localized around $\vec{r}=( \pm a, 0,0)$. In the following, we consider this case and use these states as basis states to define qubits, i.e., qubits are realized by the spin state of an electron in either orbital $|A\rangle$, or orbital $|B\rangle$.

An appropriate basis set for the six-dimensional twoparticle Hilbert space is given (using standard notation) by the three spin singlets

$$
\begin{aligned}
& \left|S_{1}\right\rangle=\frac{1}{\sqrt{2}}\left(c_{A \uparrow}^{+} c_{B \downarrow}^{+}-c_{A \downarrow}^{+} c_{B \uparrow}^{+}\right)|0\rangle, \\
& \left|S_{2}\right\rangle=\frac{1}{\sqrt{2}}\left(c_{A \uparrow}^{+} c_{A \downarrow}^{+}+c_{B \uparrow}^{+} c_{B \downarrow}^{+}\right)|0\rangle, \\
& \left|S_{3}\right\rangle=\frac{1}{\sqrt{2}}\left(c_{A \uparrow}^{+} c_{A \downarrow}^{+}-c_{B \uparrow}^{+} c_{B \downarrow}^{+}\right)|0\rangle,
\end{aligned}
$$

and the triplet multiplet,

$$
\begin{gathered}
\left|T^{-1}\right\rangle=c_{A \downarrow}^{+} c_{B \downarrow}^{+}|0\rangle, \\
\left|T^{0}\right\rangle=\frac{1}{\sqrt{2}}\left(c_{A \uparrow}^{+} c_{B \downarrow}^{+}+c_{A \downarrow}^{+} c_{B \uparrow}^{+}\right)|0\rangle, \\
\left|T^{1}\right\rangle=c_{A \uparrow}^{+} c_{B \uparrow}^{+}|0\rangle .
\end{gathered}
$$

The three triplet states are degenerate (typically, we can ignore possible Zeeman splittings ${ }^{6}$ ) and have the common eigenvalue,

$$
\varepsilon_{T}=2 \varepsilon+V_{-},
$$

where we have defined

$$
\varepsilon=\langle A|h| A\rangle=\langle B|h| B\rangle
$$

and

$$
V_{-}=\left\langle T^{\alpha}|C| T^{\alpha}\right\rangle, \quad V_{+}=\left\langle S_{1}|C| S_{1}\right\rangle .
$$

An important further observation is that, as a consequence of inversion symmetry along the axis connecting the dots, the Hamiltonian does not have any nonzero matrix elements between the singlet state $\left|S_{3}\right\rangle$ and other states. Hence, $\left|S_{3}\right\rangle$ is, independently of the system parameters, an eigenstate. The eigenvalues of the triplet and $\left|S_{3}\right\rangle$, however, do depend on system parameters. The Hamiltonian acting on the remaining space spanned by $\left|S_{1}\right\rangle$ and $\left|S_{2}\right\rangle$ can be written as

$$
\mathcal{H}=2 \varepsilon+\frac{1}{2} U_{H}+V_{+}-\left(\begin{array}{cc}
U_{H} / 2 & 2 t_{H} \\
2 t_{H} & -U_{H} / 2
\end{array}\right),
$$

where

$$
t_{H}=\langle A|h| B\rangle=\langle B|h| A\rangle
$$

and

$$
U_{H}=\left\langle S_{2}|C| S_{2}\right\rangle-V_{+} .
$$

The nontrivial part of Eq. (15) is a simple Hubbard Hamiltonian and can be identified as the Hamiltonian of a pseudospin-half object in a pseudomagnetic field having a component $U_{H}$ in the $\hat{z}$ direction and $4 t_{H}$ in the $\hat{x}$ direction of pseudospin space. (Note that this pseudospin is not related to the spin degree of freedom that provides the qubit) The space spanned by $\left|S_{1}\right\rangle$ and $\left|S_{2}\right\rangle$ contains the ground state of the system. The basis states themselves are eigenstates only in the case of a vanishing tunneling amplitude $t_{H}$ where $\left|S_{1}\right\rangle$ 
is the ground state. In all other cases, the ground state has an admixture of double occupied states contained in $\left|S_{2}\right\rangle$. The energy gap between the triplet and the singlet ground state is

$$
\varepsilon_{T}-\varepsilon_{S 0}=V_{+}-V_{-}-\frac{U_{H}}{2}+\frac{1}{2} \sqrt{U_{H}^{2}+16 t_{H}^{2}} .
$$

A swap operation in the present system is a unitary transformation that turns a state having the qubits in different states, say,

$$
c_{A \uparrow}^{+} c_{B \downarrow}^{+}|0\rangle=\frac{1}{\sqrt{2}}\left(\left|T^{0}\right\rangle+\left|S_{1}\right\rangle\right),
$$

into a state where the contents of the qubits are interchanged,

$$
c_{A \downarrow}^{+} c_{B \uparrow}^{+}|0\rangle=\frac{1}{\sqrt{2}}\left(\left|T^{0}\right\rangle-\left|S_{1}\right\rangle\right) .
$$

These two states are eigenstates in the case $V_{+}=V_{-}$and $t_{H}=0$ for which the singlet-triplet splitting vanishes.

As discussed in Refs. 2 and 6, swapping may be achieved by the action of a gate that lowers the potential barrier between the quantum dots. This leads to exponentially larger values for both $V_{+}-V_{-}$and $t_{H}$. It is adequate for our purposes to consider a model where $V_{+}=V_{-}$(consistent with the above limit of small overlap $S$ ) and the singlet-triplet splitting results entirely from $t_{H}$. If the duration and amplitude of a tunneling pulse is adjusted appropriately, the relative phase between the singlet and the triplet state involved picks up a shift of $\pi$, and a swapping operation is performed.

As pointed out in Ref. 10, a finite tunneling amplitude necessarily leads to a finite probability for double occupancies of qubit states. If double occupancy errors occur to any sizable extent as a result of the swapping process, any quantum computation based on this hardware is likely to fail. However, if the double occupancies are sufficiently rare after the swapping process, errors in the quantum computation can likely be corrected dynamically. An important observation is that the double-occupancy probability after the swap vanishes in the adiabatic limit, i.e., if the ramp time $\tau$ of the quantum gate is such that $\hbar / \tau$ is much larger than the pseudospin splitting $\sqrt{U_{H}+16 t_{H}^{2}}$. This follows since the nonadiabatic effects can arise only from the states $\left|S_{1}\right\rangle$ and $\left|S_{2}\right\rangle$, which have a nontrivial time evolution when the tunneling amplitude $t_{H}$ is time dependent. Thus, the question of whether double occupancies are problematic for swap operations in the present system is reduced to the question of how close the motion of a spin-half object in a time-dependent magnetic field is to its adiabatic limit. This will be investigated further in Sec. IV.

The reduction of the dynamics to the time evolution of a two-level system relies on the fact that the system has inversion symmetry along the $\hat{x}$ axis in real space connecting the dots. This symmetry can be broken if odd powers of the particle coordinates $x_{i}$ are added to the Hamiltonian (1), for example, the potential of a homogeneous electric field. However, the only additional matrix element due to such terms in the Hamiltonian occurs in the subspace of double-occupied states between the singlets $\left|S_{2}\right\rangle$ and $\left|S_{3}\right\rangle$. Thus, in the presence of an electric field $\mathcal{E}=-e E \Sigma_{i} x_{i}$, the Hamitonian acting on the singlet subspace spanned by $\left|S_{1}\right\rangle,\left|S_{2}\right\rangle,\left|S_{3}\right\rangle$ reads

$$
\mathcal{H}=2 \varepsilon+\frac{1}{2} U_{H}+V_{+}-\left(\begin{array}{ccc}
U_{H} / 2 & 2 t_{H} & 0 \\
2 t_{H} & -U_{H} / 2 & F \\
0 & F & -U_{H} / 2+2 X
\end{array}\right)
$$

with the real matrix element $F=\left\langle S_{2}|\mathcal{E}| S_{3}\right\rangle$ and

$$
2 X=\left\langle S_{2}|C| S_{2}\right\rangle-\left\langle S_{3}|C| S_{3}\right\rangle=2\langle A|\langle A|C| B\rangle| B\rangle .
$$

With a finite matrix element $F$, the dynamics of the system is slightly more complicated, but also in this case the only coupling of the two-qubit states (19) and (20) to the subspace of double-occupied states is provided by the tunneling amplitude $t_{H}$. Therefore, with respect to the adiabaticity of the swapping process, the situation can be expected to be not very different from the one with inversion symmetry between the dots. This will be verified in Sec. IV.

So far we have not considered a possible Zeeman coupling to the electron spin. This would not change the situation essentially since all states involved in the swapping process $\left(\left|T^{0}\right\rangle,\left|S_{1}\right\rangle,\left|S_{2}\right\rangle\right.$, and eventually $\left.\left|S_{3}\right\rangle\right)$ have the total spin quantum number $S^{z}=0$.

\section{ENTANGLED STATES}

Before analyzing further the possibility of performing swap operations in the above system, let us introduce an appropriate measure for the entanglement of its quantum states. Consider a system of two fermions living in a fourdimensional one-particle space. A general state vector in this six-dimensional Hilbert space can be written as

$$
|w\rangle=w_{a b} c_{a}^{+} c_{b}^{+}|0\rangle
$$

where a summation convention is understood for repeated latin indices $a, b, \ldots, \in\{1,2,3,4\}$ running over the orthonormalized one-particle states. The coefficient matrix $w$ can be assumed to be antisymmetric, $w_{a b}=-w_{b a}$. The normalization condition reads

$$
\langle w \mid w\rangle=1 \Leftrightarrow \operatorname{Tr}(\bar{w} w)=-\frac{1}{2},
$$

where the bar denotes complex conjugation. A two-particle state of the form (23) is, in general, entangled, i.e., it cannot be written as a single Slater determinant. $|w\rangle$ is nonentangled, i.e., a single Slater determinant, if $w$ has the form

$$
w_{a b}=\frac{1}{2}\left(z_{a}^{1} z_{b}^{2}-z_{b}^{1} z_{a}^{2}\right)
$$

for two orthonormal spinors $z^{1}$ and $z^{2}, z_{a}^{i} z_{a}^{j}=\delta^{i j}$. We note that for a given nonentangled state $|w\rangle$ the choice of spinors $z_{1}$ and $z_{2}$ is by no means unique since any $\mathrm{SU}(2)$ transformation among these two occupied one-particle states leads to 
the same two-particle state vector $|w\rangle$. Hence, for a given nonentangled state $|w\rangle$ there is a three-dimensional manifold of spinors fulfilling Eq. (25).

We define the dual matrix $\tilde{w}$ of $w$ by

$$
\tilde{w}_{a b}=\frac{1}{2} \varepsilon^{a b c d} \bar{w}_{c d}
$$

with $\varepsilon^{a b c d}$ denoting the totally antisymmetric unit tensor in four dimensions. The scalar product of a state $|w\rangle$ with its dual state $|\tilde{w}\rangle$ can be written as

$$
\langle\tilde{w} \mid w\rangle=\varepsilon^{a b c d} w_{a b} w_{c d}=8\left(w_{12} w_{34}+w_{13} w_{42}+w_{14} w_{23}\right) .
$$

This cyclic sum vanishs identically if $w$ has the form (25). Hence, the vanishing of

$$
\eta(w):=|\langle\tilde{w} \mid w\rangle|
$$

is a necessary condition for $|w\rangle$ being a single Slater determinant. Moreover, in the Appendix it is shown that $\eta(w)$ $=0$ is actually also a suffcient condition for $|w\rangle$ being nonentangled. Thus, $\eta$ defines a measure of entanglement that is exactly zero for nonentangled states. $\eta(w) \neq 0$ is therefore a necessary and sufficient condition for entanglement of quantum states. Maximally entangled states are characterized by the fact that they are collinear with their dual states $\eta(w)$ $=1$. As simple examples, we consider the basis states used in the preceding section: the states $\left|T^{-1}\right\rangle$ and $\left|T^{1}\right\rangle$ are single Slater determinants, while all other basis states are maximally entangled.

The matrix $w$ transforms under a unitary transformation of the one-particle space,

$$
c_{a}^{+} \mapsto \mathcal{U} c_{a}^{+} \mathcal{U}^{+}=U_{b a} c_{b}^{+},
$$

as

$$
w \mapsto U w U^{T},
$$

where $U^{T}$ is the transpose (not the adjoint) of $U$. It is straightforward to see that $\eta$ is invariant under such transformations, and the determinant of $w$ remains the same up to a possible phase factor. Thus, the entanglement of a state $|w\rangle$ quantified by $\eta$ does not depend on the basis chosen for the one-particle space, which is of course a necessary requirement for a measure of entanglement.

The dualization of a state can be identified as a particlehole transformation,

$$
\mathcal{U}_{p-h} c_{a}^{+} \mathcal{U}_{p-h}^{+}=c_{a}, \quad \mathcal{U}_{p-h}|0\rangle=c_{1}^{+} c_{2}^{+} c_{3}^{+} c_{4}^{+}|0\rangle,
$$

followed by complex conjugation. In fact,

$$
\mathcal{U}_{p-h}|w\rangle=-|\overline{\tilde{w}}\rangle \text {. }
$$

We note that the complex conjugations in Eqs. (26) and (32) are unimportant for states $|w\rangle$ such that $\eta(w)=0$, since a single Slater determinant is always orthogonal to its particlehole conjugate, irrespective of a possible phase transformation of a prefactor. However, the complex conjugation in the definition (26) is essential to the sufficiency of the above condition. As an example, consider a state $|w\rangle$ with $w_{12}$ $=w_{34}=1 / 4, \quad w_{13}=w_{24}=i / 4$, and $w_{14}=w_{23}=0$. This is clearly a maximally entangled state, $\eta(w)=1$, while its scalar product with the complex conjugate of its dual state is $\langle\tilde{\tilde{w}} \mid w\rangle=0$.

We also mention the following identity for the determinant of $w$ :

$$
\operatorname{det} w=\left(\frac{1}{8}\langle\tilde{w} \mid w\rangle\right)^{2}
$$

Hence it follows that also $|\operatorname{det} w|$ could be used as a measure of entaglement. Equation (33) is important for the proof of the sufficiency of our criterion for nonentangled states, as explained in detail in the Appendix.

A convenient choice to make contact between the general state labels $a, b, \ldots \in\{1,2,3,4\}$ used here and the basis states of the preceding section is given by $(1,2,3,4)$ $=(A \uparrow, A \downarrow, B \uparrow, B \downarrow)$. With this convention, a state vector spanned by $\left|S_{2}\right\rangle$ and $\left|S_{3}\right\rangle$ only has $w_{12}$ and $w_{34}$ as its only independent nonzero coefficients in $w$. Such a state lies fully in the subspace of double occupancies, and its entanglement is purely due to the orbital degrees of freedom

$$
\eta_{\text {orb }}=8\left|w_{12} w_{34}\right| .
$$

On the other hand, a state spanned by $\left|S_{1}\right\rangle$ and $\left|T^{0}\right\rangle$ has no double occupancies and is entangled purely with respect to the spin degrees of freedom,

$$
\eta_{\text {spin }}=8\left|w_{14} w_{23}\right| .
$$

For a general state vector, both kinds of entanglement (orbital and spin) contribute to $\eta(w)$.

\section{RESULTS FOR THE SWAPPING PROCESS}

We now continue with our investigation of the dynamics of the double quantum dot qubit swapping process generated by a time-dependent tunneling amplitude.

Let us first consider the case of inversion symmetry along the axis connecting the dots. As explained in Sec. II, this problem can be reduced essentially to the time evolution of a pseudospin-half object in a magnetic field having a timedependent component in the $x$ direction of the pseudospin space. In the course of swapping, the triplet contribution to the incoming state (19) will just pick up a phase factor according to its constant eigenvalue, while the singlet contribution will mix with the other singlet $\left|S_{2}\right\rangle$. Therefore, a finite probability for double occupancies will necessarily arise $d u r$ ing the swapping process. However, if these amplitudes can be suppressed sufficiently when the swapping is complete (as in the adiabatic limit) errors in the quantum computation can be avoided. Thus, we are left with the question of how close the dynamics of our formal spin-half object is to its adiabatic limit. We note that, in the adiabatic limit, no Berry phase occurs in the time evolution of the singlet states, since the motion of the formal spin is restricted to a plane. Hence, the solid angle encircled in a round trip is strictly zero.

The integration of the Schrödinger equation for our timedependent two-level problem is, in general, nonelementary. 


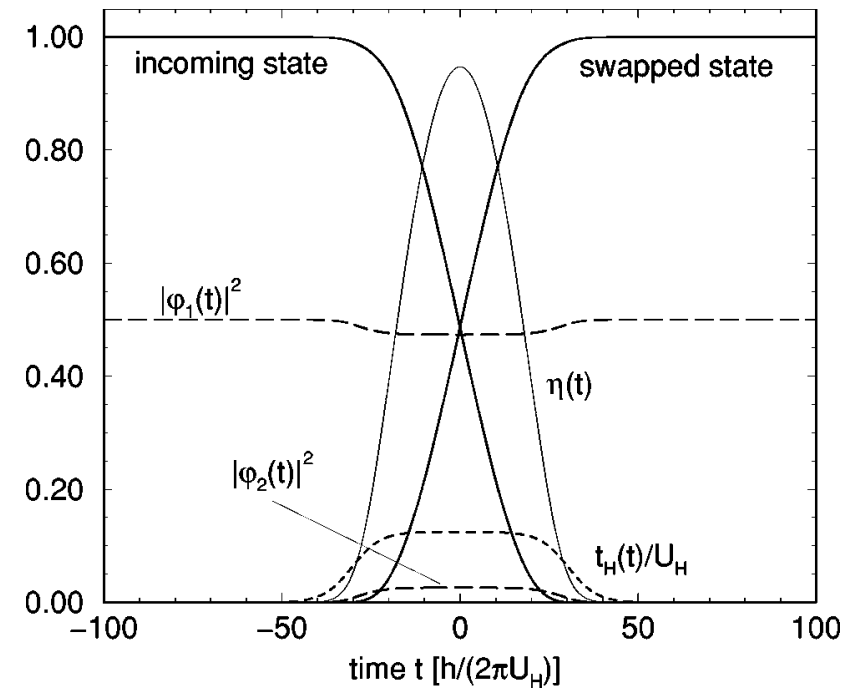

FIG. 1. A swap process as a function of time. The tunneling amplitude $t_{H}(t)$ is plotted (in units of $U_{H}$ ) as a dotted line. The square amplitude of the incoming state (19) and the outgoing state (20) are shown as thick lines. The square amplitudes of the singlets $\left|S_{1}\right\rangle$ and $\left|S_{2}\right\rangle$ are denoted by $\left|\varphi_{1}\right|^{2}$ and $\left|\varphi_{2}\right|^{2}$, respectively, and plotted as long-dashed lines. The measure of entanglement $\eta(t)$ is also shown.

However, there is a considerable body of literature, starting with early work by Landau, ${ }^{13}$ Zener, ${ }^{14}$ and Rosen and Zener, ${ }^{15}$ where particular cases of this problem were reduced to well-known differential equations of mathematical physics such as the hypergeometric equation. This work was reviewed and generalized very recently in Ref. 16. However, such an approach still works only for special time-dependent Hamiltonians, i.e., in the present context, only for special shapes of the tunneling pulse $t_{H}(t)$ and many quantities of interest are given by complicated nonelementary expressions that require numerical evaluation. For this reason and for the sake of brevity of our paper, we shall resort to numerical integrations of the Schrödinger equation. From such studies we will see that the range of adiabaticity is remarkably large. Our numerical findings will be corroborated and made physically plausible by well-known analytical results for LandauZener-type transitions in simplified cases.

To be specific, we consider a time-dependent tunneling of the form

$$
t_{H}(t)=\frac{\Delta}{1+\frac{\cosh (t / \tau)}{\cosh [T /(2 \tau)]}} .
$$

This is a tunneling pulse that is switched on and off exponentially with a characteristic time $\tau$. It has a duration of $T$ and an amplitude given by $\Delta$ (for $T \gg \tau$ ). Therefore, this form is flexible enough to describe the essential features of a pulse. The exponential switching is motivated by the exponential-like dependence of the tunneling matrix element on external parameters. ${ }^{6}$

A typical situation is shown in Fig. 1 for a switching time of $\tau=4 \hbar / U_{H}$, an amplitude of $\Delta=U_{H} / 8$, and the duration $T$ adjusted to enable single swap operation. The figure shows the results of a numerical integration of the time-dependent Schrödinger equation using the fourth-order Runge-Kutta scheme. The time-dependent tunneling amplitude $t_{H}(t)$ is plotted (in units $U_{H}$ ) as a dotted line. The square amplitude of the incoming state (19) and the outgoing state (20) are shown as thick lines. The square amplitudes of the singlets $\left|S_{1}\right\rangle$ and $\left|S_{2}\right\rangle$ are denoted by $\left|\varphi_{1}\right|^{2}$ and $\left|\varphi_{2}\right|^{2}$, respectively, and plotted as long-dashed lines. The probability of double occupancies is given by $\left|\varphi_{2}\right|^{2}$. As one can see from Fig. 1, this quanity is finite during the swapping process but strongly suppressed afterwards. The measure of entanglement $\eta(t)$ is also shown Fig. 1. It is zero for the nonentangled incoming and outgoing state, and achieves its maximum value of almost unity in the middle of the process. This quantifies and shows explicitly the entanglement of the quantum state during the swapping process.

The probability $\left|\varphi_{2}\right|^{2}$ for double occupancy after switching off the tunneling depends on the switching time $\tau$, the amplitude $\Delta$, and also on the duration $T$ of the tunneling pulse, i.e., on the exact time when the switching off sets in. However, our numerics suggest that there is an upper bound for $\left|\varphi_{2}\right|^{2}$ at given $\tau$ and $\Delta$. In the above example, the double occupancy probability after the swapping process is smaller than $10^{-10}$, which is a very tiny value. A typical order of magnitude for the double occupancy probability is $10^{-6}$ for amplitudes $\Delta<U_{H}$ and switching times $\tau>4 \hbar / U_{H}$. In fact, also larger values of $\Delta$ (being still comparable with $U_{H}$ ) can be possible, leading to double occupancy probabilities of the same order, while this probability significantly increases if $\tau$ becomes smaller than $4 \hbar / U_{H}$. Thus, this value characterizes the region where the motion of the system is close to its adiabatic limit and is remarkably small on the natural time scale of the system given by $\hbar / U_{H}$, while adiabatic behavior is, in general, expected for a particularly slow time evolution.

This large range of quasiadiabatic behavior can be understood qualitatively by considering a simplified situation where the tunneling is switched on and off linearly in time and is constant otherwise. Then, nonadiabtic effects can occur only during the sharply defined switching processes. For simplicity, we consider the first switching process only where the tunneling has the time dependence $t_{H}=(\Delta / \tau), t$ $\in[0, \tau]$. To enable analytical progress, let us further assume $t \in[-\infty, \infty]$, which should lead to an upper bound for the probability of nonadiabatic transitions due to the switching. This problem was considered a long time ago by Landau ${ }^{13}$ and by Zener. ${ }^{14}$ The result of Ref. 14 for the probability of nonadiabatic transitions reads

$$
P_{\text {nad }}=e^{-\alpha}
$$

with an adiabaticity parameter

$$
\alpha=\frac{\pi}{8} \frac{U_{H}^{2}}{\hbar(\Delta / \tau)} .
$$

We see that the probability for nonadiabatic transitions is exponentially suppressed with increasing switching time $\tau$. This exponential dependence explains qualitatively the 


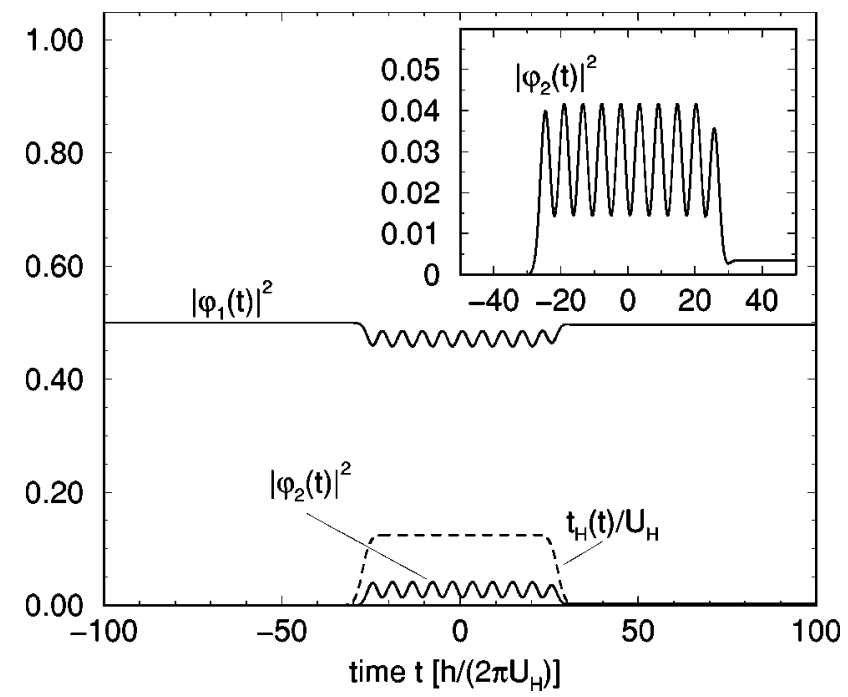

FIG. 2. The square amplitudes of the singlet states $\left|S_{1}\right\rangle$ and $\left|S_{2}\right\rangle$ for the same situation as in Fig. 1, but with a four-times smaller ramp time of only $\tau=\hbar / U_{H}$. The inset shows $\left|\varphi_{2}(t)\right|^{2}$ on a magnified scale. The dynamics of the system is clearly in the nonadiabatic regime.

above observation of a large range of quasiadiabatic bahavior. To obtain an estimate for a nonlinear switching one may replace the ratio $(\Delta / \tau)$ in the denominator of Eq. (38) by the maximum time derivative of the tunneling $t_{H}(t)$ [giving $\alpha$ $=\pi U_{H}^{2} / 3 \hbar(\Delta / \tau)$ for the pulse (36)].

A similar exponential dependence of the probability for nonadiabatic transitions on the switching time $\tau$ was also found analytically by Rosen and Zener ${ }^{15}$ for a particular twoparametric pulse of the form

$$
t_{H}(t)=\Delta / \cosh (t / \tau)
$$

In this case, nonadiabatic transitions occur with a probability

$$
P_{\text {nad }}=\sin ^{2}[\Delta \tau /(2 \hbar)] / \cosh ^{2}\left[U_{H} \tau /(2 \hbar)\right] .
$$

To illustrate the behavior in the strongly nonadiabatic case, we have plotted in Fig. $2\left|\varphi_{1}\right|^{2}$ and $\left|\varphi_{2}\right|^{2}$ for the same situation as in Fig. 1, but with a four times smaller ramp time of only $\tau=\hbar / U_{H}$. In this case, small oscillations occur in the time evolution of these two quantities during the tunneling pulse, which can be understood in terms of the eigenspectrum at a given tunneling $t_{H}=\Delta$. Additionally, a sizable double occupancy probability of about 0.005 remains after the pulse, as shown in the inset.

Figure 3 shows a square root of a swap, which is obtained from the situation of Fig. 1 by halving the duration $T$ of the tunneling pulse. The resulting state is a fully entangled complex linear combination of the states $\left|S_{1}\right\rangle$ and $\left|T^{0}\right\rangle$, or, equivalently, of the incoming state (19) and the outgoing state (20) of the full swap. Again, the weight of the doubly occupied state $\left|S_{2}\right\rangle$ is strongly suppressed after the tunneling pulse. As a consequence, Eq. (34) implies that $\eta_{\text {orb }}=0$ after the completion of switching, while $\eta=\eta_{\text {spin }}=8\left|w_{14} w_{23}\right|$

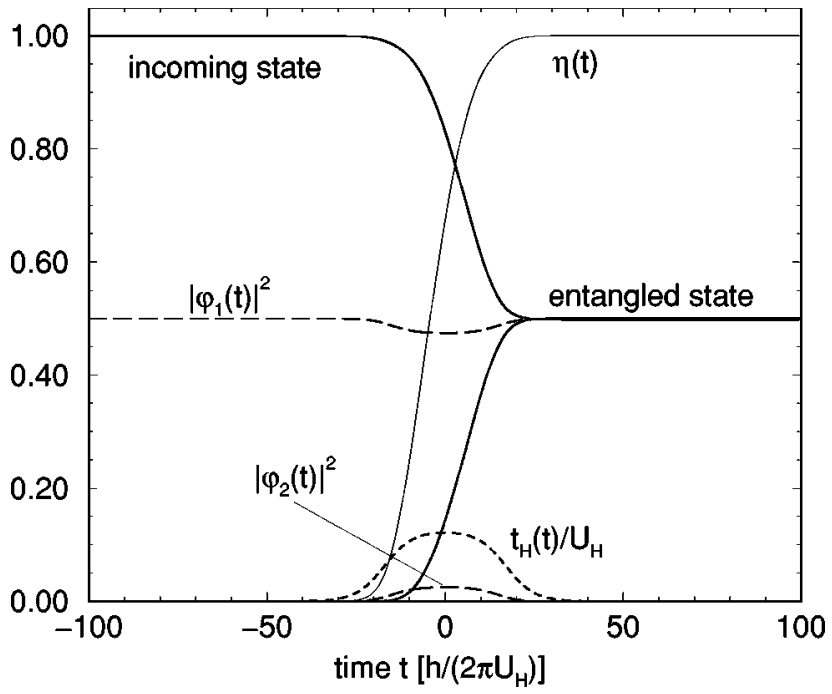

FIG. 3. A square root of a swap, which is obtained from the situation of Fig. 1 by halving the pulse duration $T$. The probability of double occupancies is again strongly suppressed after the tunneling pulse. The resulting state is a fully entangled complex linear combination of $\left|S_{1}\right\rangle$ and $\left|T^{0}\right\rangle$, or, equivalently, of the incoming state (19) and the outgoing state (20) of the full swap. The quantum mechanical weights of the latter states are plotted as thick solid lines.

$=1$. This shows that the entanglement of the two electrons is entirely in the spin (and not in the orbital) degrees of freedom after switching.

Let us finally consider swapping processes when the inversion symmetry along the axis connecting the dots is broken. Such processes are governed by the Hamiltonian (21) in the presence of a finite matrix element $F$. Our numerical results are, in this case, qualitatively the same as before with the admissible switching times $\tau$ slightly growing with increasing $F$. In Fig. 4 we illustrate our findings for a comparatively large off-diagonal element $F=0.4 U_{H}$. The additional Coulomb matrix element is $X=0.2 U_{H}$, and the parameters of the tunneling pulse are $\tau=8 \hbar / U_{H}$ and $\Delta=U_{H} / 8$ with a duration $T$ appropriate for a single swapping. As a result, a clean swapping operation can be performed also in the absence of inversion symmetry.

We note that the Hund-Mulliken scheme used here is restricted to the low-energy sector where only the lowest single-particle energy levels (with typical spacings $\delta \epsilon$ ) are kept. For this scheme to be valid also in a switching process, we need to require that time-dependent changes must be performed adiabatically also with respect to the time scale set by $\hbar / \delta \epsilon$, i.e., we need $\tau>\hbar / \delta \epsilon{ }^{6}$ On the other hand, to suppress double-occupancy errors we have seen that the adiabaticity parameter $\alpha$ of Eq. (38) must exceed one, implying that $\tau>8 \hbar \Delta /\left(\pi U_{H}^{2}\right)$. Thus, the adiabaticity condition for switching becomes more generally,

$$
\tau>\tau_{\min }:=\max \left\{\frac{\hbar}{\delta \epsilon}, \frac{8 \hbar}{\pi} \frac{\Delta}{U_{H}^{2}}\right\} .
$$

There are now two particular cases we can distinguish. First, if the effective Coulomb charging energy exceeds the level 


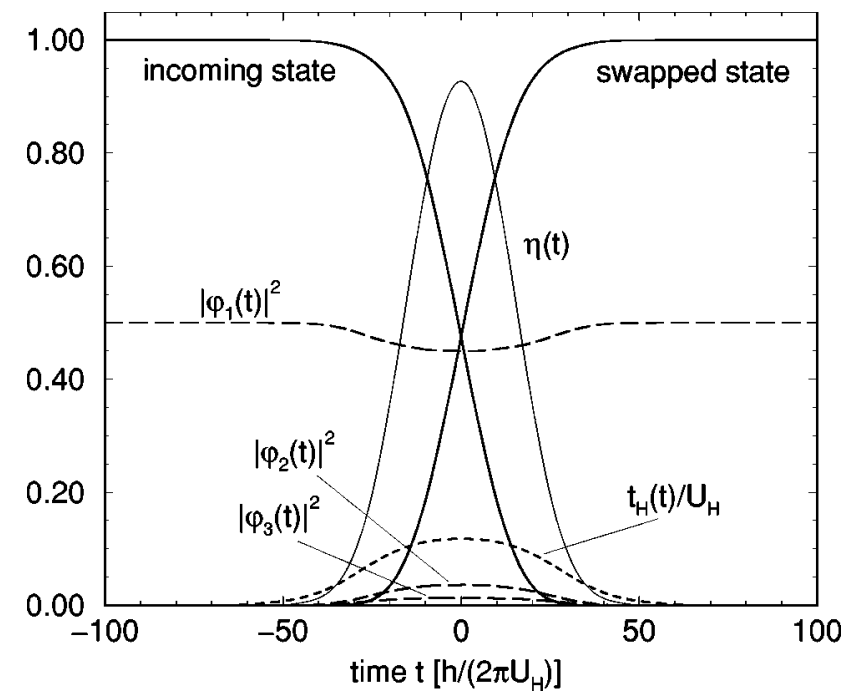

FIG. 4. A swapping processes in the absence of inversion symmetry along the axis connecting the dots. The square amplitudes of the singlet states $\left|S_{i}\right\rangle, i \in\{1,2,3\}$, are denoted by $\left|\varphi_{i}\right|^{2}$. The additional matrix elements entering the Hamiltonian (21) are $X$ $=0.2 U_{H}$ and $F=0.4 U_{H}$. The parameters of the tunneling pulse are $\tau=8 \hbar / U_{H}$ and $\Delta=U_{H} / 8$ with a duration $T$ appropriate for a single swapping. As a result, a clean swapping operation can be performed also in the absence of inversion symmetry.

spacing, i.e., $U_{H}>\delta \epsilon$, we obtain $\tau_{\min }=\hbar / \delta \epsilon$, since for consistency we have $\Delta<\delta \epsilon$. Thus, when the switching is adiabatic with respect to the scale set by $\delta \epsilon$, errors due to double occupancy are automatically excluded. In the second case with $U_{H}<\sqrt{\Delta \delta \epsilon}<\delta \epsilon$ ("ultrasmall quantum dots") we obtain $\tau_{\min }=8 \hbar \Delta /\left(\pi U_{H}^{2}\right)$, which means that the overall condition for adiabaticity is determined by the no-double occupancy criterion. Using typical material parameters for GaAs quantum dots, ${ }^{17}$ we can estimate ${ }^{6}$ that $\tau_{\min }$ is of the order of $50 \mathrm{ps}$.

\section{CONCLUSIONS}

We have studied a double quantum dot system as a quantum gate swapping the electronic spin states on the two dots. Within the Hund-Mulliken approach, the dynamics of such a system having inversion symmetry along the axis connecting the dots reduces to the problem of a pseudospin-half object in a time-dependent pseudomagnetic field. By solving the time-dependent Schrödinger equation numerically we demonstrate the possibility of performing swap operations and investigate the role of double occupancies of the dots. These double occupancies are found to be (exponentially) strongly reduced, as a result of the swapping process, for a large range of system parameters and are therefore not a principle obstacle for quantum computation in such systems. Further numerical studies show that this situation is not altered qualitatively when the inversion symmetry is broken.

Moreover, we have introduced an appropriate measure of entanglement that takes explicitly into account the possibility of double occupancies. This quantity allows to quantify the entanglement of the quantum state during a gate operation and provides a necessary and sufficient condition for entangled states. Hence, we expect this measure of entanglement to be useful in general in the study of quantum information phenomena in systems such as (real or artificial) diatomic molecules, or other quantum-confined two-site structures.

\section{ACKNOWLEDGMENTS}

We thank Guido Burkard for useful discussions and comments on this paper. J.S. was supported by the Deutsche Forschungsgemeinschaft under Grant No. Schl 539/1-1 and acknowledges the hospitality of the Institute for Theoretical Physics of Hannover University, Germany, where this work was completed. D.L. acknowledges partial support from the Swiss National Science Foundation. A.H.M. acknowledges support from the National Science Foundation under Grant No. DMR-9714055.

\section{APPENDIX}

Here we give the proof that $\eta(w)=0$ is indeed a sufficient condition for $|w\rangle$ being a single Slater determinant state. The proof consists of two steps.

(i) Let $w$ be purely real. Since $\eta(w)=0$ implies det $w$ $=0$ [cf. Eq. (33)] $w$ has at least one zero eigenvalue. Because $w$ is real and antisymmetric its eigenvalues are purely imaginary (if not zero) and occur in pairs of complex conjugates. Therefore, at least two of the four eigenvalues of $w$ are zero. It follows from standard arguments (similar to those for real and symmetric matrices) that these two zero eigenvalues correspond to two real eigenvectors being orthogonal onto each other. It follows that there is a real and orthogonal oneparticle transformation $U$ so that, say, the first two rows and columns of the resulting matrix $U w U^{T}$ are zero. Hence, the one-particle states with labels $a=1,2$ (in this new basis) are strictly empty, and the two electrons occupy the remaining two states. Thus, $|w\rangle$ is clearly a single Slater determinant.

(ii) General case: w complex. By a one-particle transformation $\mathcal{U}$ with

$$
U=\operatorname{diag}\left(e^{i \phi_{1}}, e^{i \phi_{2}}, e^{i \phi_{3}}, e^{i \phi_{4}}\right),
$$

one can adjust the phases in $w^{\prime}=U w U^{T}$ in a manner that, say, $w_{12}^{\prime}, w_{13}^{\prime}, w_{14}^{\prime}$ are real. Denoting the real and imaginary part of $w^{\prime}$ by

$$
w^{\prime}=u+i v
$$

it follows that $\operatorname{det} v=0$. Consider now the (unnormalized) states $|u\rangle$ and $|v\rangle$. If one of these states vanishes the assertion is already proved in (i), thus assume $|u\rangle \neq 0 \neq|v\rangle$. The condition $\eta(w)=0$ reads

$$
\langle\tilde{u} \mid u\rangle-\langle\tilde{v} \mid v\rangle+i(\langle\tilde{u} \mid v\rangle+\langle\tilde{v} \mid u\rangle)=0 .
$$

Since both terms in the imaginary part are equal by definition and $\operatorname{det} v=0$ implies $\langle\tilde{v} \mid v\rangle=0$, it holds

$$
\langle\tilde{u} \mid u\rangle=0 \Rightarrow \operatorname{det} u=0
$$


and

$$
\langle\tilde{u} \mid v\rangle=\langle\tilde{v} \mid u\rangle=0
$$

From (i) one concludes that both $|u\rangle$ and $|v\rangle$ are single Slater determinants. Thus, there are spinors $x^{1}, x^{2}$ and $y^{1}, y^{2}$ with

$$
u_{a b}=\frac{1}{2}\left(x_{a}^{1} x_{b}^{2}-x_{b}^{1} x_{a}^{2}\right), \quad v_{a b}=\frac{1}{2}\left(y_{a}^{1} y_{b}^{2}-y_{b}^{1} y_{a}^{2}\right) .
$$

Moreover, Eq. (A5) implies that

$$
\varepsilon^{a b c d} x_{a}^{1} x_{b}^{2} y_{c}^{1} y_{d}^{2}=0 .
$$

Thus, the $4 \times 4$ matrix having these four spinors as its rows or columns has a vanishing determinant. Therefore these spinors are linearly dependent. Without loss of generality, consider the case

$$
x^{1}=\alpha x^{2}+\beta y^{1}+\gamma y^{2},
$$

where the complex coefficients $\beta$ and $\gamma$ are not both zero since otherwise $u=0$. Let, again without loss of generality, $\beta$ be nonzero. Then the spinors

$$
z^{1}=\beta y^{1}+\gamma y^{2}, \quad z^{2}=x^{2}+\frac{i}{\beta} y^{2}
$$

solve the problem, i.e.,

$$
w_{a b}^{\prime}=u_{a b}+i v_{a b}=\frac{1}{2}\left(z_{a}^{1} z_{b}^{2}-z_{b}^{1} z_{a}^{2}\right) .
$$

$z^{1}$ and $z^{2}$ are both nonzero and not collinear to each other since otherwise $w^{\prime}=0$. Thus, up to an unimportant orthonormalization, these two spinors define one-particle states that allow us to express $\left|w^{\prime}\right\rangle$ (and consequently $|w\rangle$ ) as a single Slater determinant.
${ }^{1}$ A. Steane, Rep. Prog. Phys. 61, 117 (1998).

${ }^{2}$ D. Loss and D. P. DiVincenzo, Phys. Rev. A 57, 120 (1998).

${ }^{3}$ J. M. Kikkawa, I. P. Smorchkowa, N. Samarth, and D. D. Awschalom, Science 277, 1284 (1997).

${ }^{4}$ J. M. Kikkawa and D. D. Awschalom, Phys. Rev. Lett. 80, 4313 (1998).

${ }^{5}$ J. A. Gupta, D. D. Awschalom, X. Peng, and A. P. Alivisatos, Phys. Rev. B 59, R10 421 (1998); 60, 8394(E) (1999).

${ }^{6}$ G. Burkard, D. Loss, and D. P. DiVincenzo, Phys. Rev. B 59, 2070 (1999).

${ }^{7}$ G. Burkard, G. Seelig, and D. Loss, Phys. Rev. B 62, 2581 (2000).

${ }^{8}$ For a recent review, see G. Burkard, H.-A. Engel, and D. Loss, Fortschr. Phys. 48, 956 (2000).

${ }^{9}$ X. Hu and S. Das Sarma, Phys. Rev. A 61, 62301 (2000).
${ }^{10}$ S. Das Sarma, J. Fabian, X. Hu, and I. Zutic, cond-mat/9912040 (unpublished).

${ }^{11}$ D. P. DiVincenzo, Phys. Rev. A 51, 1015 (1995).

${ }^{12}$ For a recent review, see M. Lewenstein, D. Bruss, J. I. Cirac, M. Kus, J. Samsonowicz, A. Sanpera, and R. Tarrach, J. Mod. Opt. 77, 2481 (2000).

${ }^{13}$ L. D. Landau, Phys. Z. Sowjetunion 2, 46 (1932).

${ }^{14}$ C. Zener, Proc. R. Soc. London, Ser. A 137, 696 (1932).

${ }^{15}$ N. Rosen and C. Zener, Phys. Rev. 40, 502 (1932).

${ }^{16}$ A. M. Ishkhanyan, J. Phys. A 33, 5539 (2000).

${ }^{17}$ L. P. Kouwenhoven, C. M. Marcus, P. L. McEuen, S. Tarucha, R. M. Westervelt, and N. S. Wingreen, in Mesoscopic Electron Transport, Vol. 365 of NATO Advanced Study Institute, Series $E$, edited by L. L. Sohn, L. P. Kouwenhoven, and G. Schön (Kluwer, Dordrecht, 1997). 